\title{
Mapeo Doppler color de las perforantes de la arteria colateral radial y su posible aplicación en la realización de colgajos de perforantes para cobertura de defectos en miembro superior
}

\section{Color Doppler mapping of the perforators of the radial collateral artery and its possible application in performing piercing flaps for upper limb defect coverage}

\author{
Carlos Eduardo Torres-Fuentes ${ }^{1}$ Alexandra Bernal ${ }^{2}$ Erica Menze ${ }^{1}$ Diego Castañeda $^{1}$ Camilo Ardila ${ }^{2}$ \\ ${ }^{1}$ Hospital de San José, Servicio de Cirugía Plástica: reconstructiva y \\ estética, Bogotá D.C, Colombia \\ ${ }^{2}$ Hospital de San José, Servicio de Radiología, Bogotá D.C, Colombia \\ Address for correspondence Carlos Eduardo Torres Fuentes, MD, Msc, \\ Hospital de San Jose, Bogotá D.C., Colombia \\ (e-mail: drtorresplastic@yahoo.com).
}

Rev Iberam Cir Mano 2019;47:91-96.

\section{Resumen \\ Palabras clave \\ - colgajo perforante \\ - extremidad superior \\ - arteria colateral radial \\ - velocidad de flujo sanguíneo \\ - ultrasonografía Doppler \\ Introducción El uso de los colgajos regionales basados en arterias perforantes musculocutáneas o fasciocutáneas, constituyen una herramienta útil en el manejo en defectos de cobertura complejos. \\ Objetivo Determinar las características anatómicas de las perforantes de la arteria colateral radial, rama de la arteria braquial profunda, en voluntarios sanos según los hallazgos en la ecografía Doppler color. \\ Métodos Se realizó un estudio descriptivo, en donde se describió el patrón de las perforantes de la arteria colateral radial (ACR) mediante ecografía Doppler color reportando diámetro, velocidad picosistólica (VPS) y presencia de vena acompañante, en una muestra de 30 voluntarios sanos que cumplieran con los criterios de inclusión. Resultados Se realizó el estudio doppler color en ambos miembros superiores en 30 voluntarios sanos. Se encontró un promedio de 3,3 vasos perforantes derechos y 3,1 vasos perforantes izquierdos para cada brazo respectivamente, siendo la mayoría musculocutáneas en su curso, de las cuales la mayoría eran distales $67 \%$ del lado derecho y $62,7 \%$ del lado izquierdo, con un diámetro promedio de $1,3 \mathrm{~mm}$ en ambos brazos, velocidad de flujo promedio de $22,9 \mathrm{~cm} / \mathrm{s}$ del lado derecho y $19,7 \mathrm{~cm} / \mathrm{s}$ del lado izquierdo, y una distancia promedio al epicóndilo lateral de $8 \mathrm{~cm}$ del lado derecho y $7,9 \mathrm{~cm}$ del lado izquierdo. \\ Conclusión Se estableció la presencia, ubicación, tipo y características anatómicas de los vasos perforantes dependiente de la arteria colateral radial con un estudio doppler color, donde se identificó su predominio en el área distal del brazo y se describió la VPS de las perforantes encontradas.}

received

April 6, 2019

accepted

September 3, 2019
DOI https://doi.org/

10.1055/s-0039-1698807. ISSN 1698-8396.
Copyright $\odot 2019$ Thieme Revinter Publicações Ltda, Rio de Janeiro, Brazil
License terms

(c) (1) $\ominus$ (5) 


\section{Introducción}

A la hora de tratar un defecto de cobertura en una zona anatómica como el miembro superior, es importante contar con un tejido adecuado que garantice la adecuada cobertura de esa zona, dada su complejidad y función. Por lo anterior, la mejor estrategia en el manejo de esa zona anatómica es el uso de colgajos, bien sean locales, regionales o libres. ${ }^{1-4}$

El uso de los colgajos regionales basados en arterias perforantes musculocutáneas o fasciocutáneas, constituyen una novedosa opción de manejo en defectos de cobertura complejos. Dentro de los colgajos regionales, el colgajo en "propela" fue descrito inicialmente por Hyakusokuen 1991 para la cobertura de defectos en pacientes con quemaduras, y se basaba en un pedículo subcutáneo y posteriormente Hallock en el 2006 describió por primera vez un colgajo basado en perforantes fasciocutáneas, con muy buenos resultados, por lo que han sido ampliamente utilizados. Teniendo en cuenta el tamaño del defecto, la forma y la función del área a cubrir, el colgajo de perforantes, ha sido considerado desde los años 90, como una buena opción de manejo en esa patología, pues cubre el área expuesta con tejido de similar textura, grosor y color, dejando una menor morbilidad del área donante. ${ }^{1,2,5-9,14-19}$

Para el manejo de defectos en fosa cubital y codo, así como otras zonas de esta región anatómica, se destaca el uso del colgajo lateral del brazo, el cual corresponde a un colgajo septofasciocutáneo basado en perforantes de la arteria colateral radial (ACR), que a su vez es rama de la arteria braquial profunda (ABP), rama de la arteria braquial (humeral). Ese colgajo está indicado para transferencia tisular local, regional y libre. Fue descrito inicialmente por Song en 1982, Cormack en 1983 y Katsarosen 1984 , quienes documentaron por primera vez sus ventajas. Ese colgajo se extiende desde la inserción distal del deltoides hasta el epicóndilo lateral. Presenta numerosas ventajas incluyendo una anatomía vascular constante representada en una rama terminal de la arteria braquial profunda (arteria colateral radial), una mínima morbilidad del área donante y una gran versatilidad en su diseño. La arteria colateral radial irriga la piel suprayacente mediante cuatro 0 cinco perforantes septocutáneas a lo largo del septo intermuscular. La perforante septocutánea más distal típicamente se ramifica aproximadamente $4 \mathrm{~cm}$ proximalmente al epicóndilo lateral $^{10,11}$ y el drenaje venoso se lleva a cabo por dos venas concomitantes.

La isla cutánea del colgajo se localiza entre la inserción distal del deltoides y el epicóndilo lateral, coincidiendo además con el eje del pedículo. Las dimensiones de la isla son de $12 \times 5 \mathrm{~cm}$ en promedio, con una longitud del pedículo que es en promedio de $6 \mathrm{~cm} .{ }^{8,10,12-15}$ Desde el punto de vista anatómico se sabe que la bifurcación de la ABP se encuentra a nivel de la inserción distal del deltoides por debajo del vientre lateral del tríceps. El eje del pedículo pasa a lo largo del septo intermuscular lateral que se localiza entre el braquial y el braquiorradialis en el lado anterior y el vientre lateral del tríceps en el lado posterior. ${ }^{10,18,19}$

Con lo anteriormente mencionado, y teniendo en cuenta que hay pocos estudios disponibles de este tipo, surge la necesidad de realizar un estudio descriptivo de las características anatómicas, comportamiento y distribución de las perforantes de la arteria colateral radial rama de la braquial profunda, mediante Doppler Color en individuos sanos de nuestra población.

Siendo el objetivo de este estudio conocer, describir y detallar las características anatómicas de dicha fuente vascular, en el diseño de colgajos, dando paso a su aplicación para la transferencia libre y colgajos de perforantes de la arteria colateral radial principalmente en defectos de cobertura de tercio medio del miembro superior, como una opción confiable, indicada, segura y predecible, asî mismo detallar la anatomía de esta fuente vascular, teniendo en cuenta el diámetro de los vasos perforantes y la velocidad de flujo, característica obtenida únicamente por el método Doppler. $^{17}$

\section{Métodos}

\section{Diseño de investigación}

\section{Estudio descriptivo}

\section{Pacientes}

Dado que el trauma es la condición que puede requerir un colgajo con mayor frecuencia y que son los pacientes jóvenes las personas que más frecuentemente presentan esa condición, se escogieron voluntarios jóvenes de forma heterogénea para identificar los objetivos del estudio, se describió el patrón de perforantes de la arteria colateral radial mediante ecografía Doppler color en 30 estudiantes voluntarios de pregrado y postgrado de la facultad de medicina de la Fundación Universitaria de Ciencias de la Salud entre Mayo del 2017 y Noviembre del 2017, que cumplieran con los criterios de inclusión, dentro de los que se encontraban que fueran estudiantes voluntarios sanos de pregrado y posgrado de la facultad de medicina, mayores de 18 años, miembros superiores indemnes y sin ningún proceso infeccioso o inflamatorio en la extremidad. Sin embargo, no se tuvo en cuenta su estatura, ni la longitud de la extremidad. En cuanto a los criterios de exclusión, fueron antecedente de cirugía mayor en miembro superior por historia clínica (definiendo cirugía mayor como cirugía en la que se hayan manipulado tejidos profundos y vasos principales), antecedente de cualquier tipo de trauma en miembros superiores en algún momento de la vida según su historia clínica, antecedente de enfermedad vascular de pequeños vasos u otras enfermedades vasculares y/o sistémicas que puedan comprometer la vasculatura (Vasculitis, Diabetes Mellitus) o antecedente de fracturas del miembro superior.

\section{Técnica y Métodos}

Cada voluntario se colocó en decúbito supino sobre la mesa de ecografía, en donde se realizó una marcación de la isla de piel del colgajo anterolateral del brazo (localizado entre la inserción distal del deltoides y el epicóndilo lateral con medidas de $12 \mathrm{~cm}$ de largo por $6 \mathrm{~cm}$ de ancho aproximadamente). Previa aplicación de gel conductor en la 


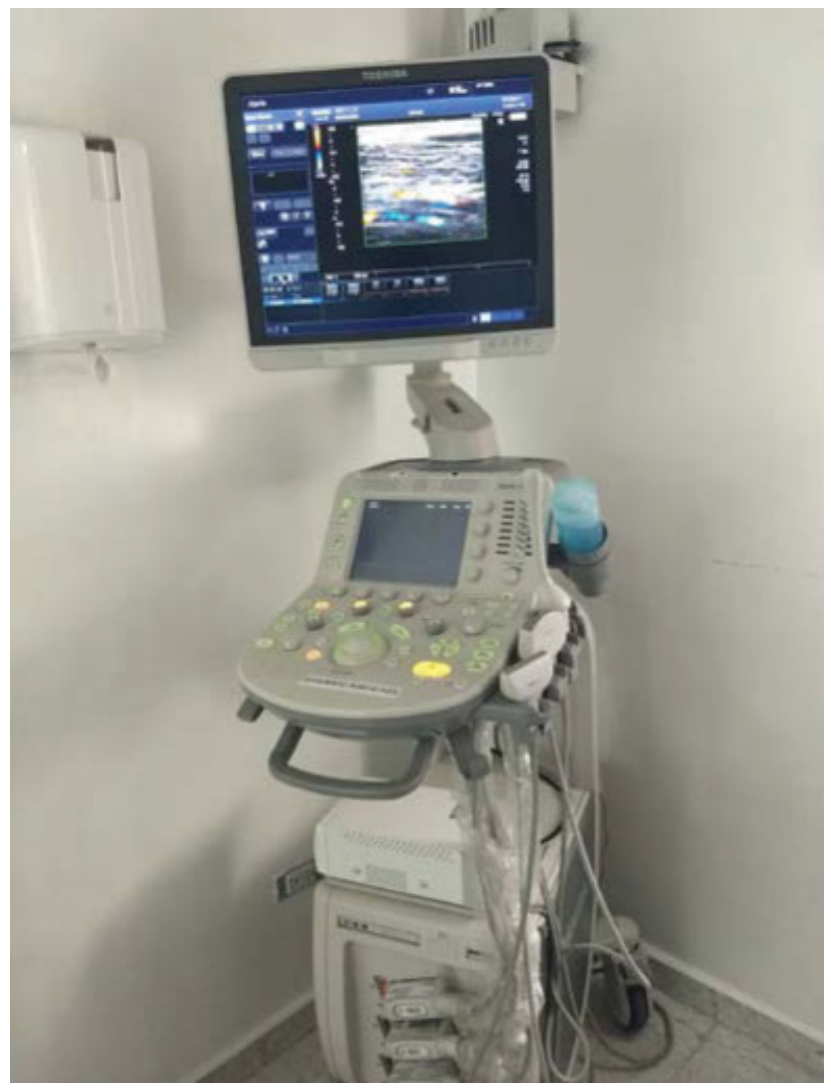

Fig. 1 Equipo de ecografía-doppler multicolor Toshiba Xario 200 utilizado para la realización del estudio.

región anterolateral de ambos brazos, seguido a ello se procedió a la valoración con un equipo de ecografía Doppler multicolor Toshiba Xario 200 (-Figura 1), el cual utiliza transductor lineal de alta frecuencia de $18 \mathrm{MHz}$ (- Figura 2), que proporciona especial detalle para los tejidos superficiales y vasculares. Fue el instructor de radiología del hospital, quien realizó la valoración y respectiva marcación de la arteria colateral radial desde el músculo deltoides, el cual se valoró desde el tercio medio en sentido transversal hasta su inserción,

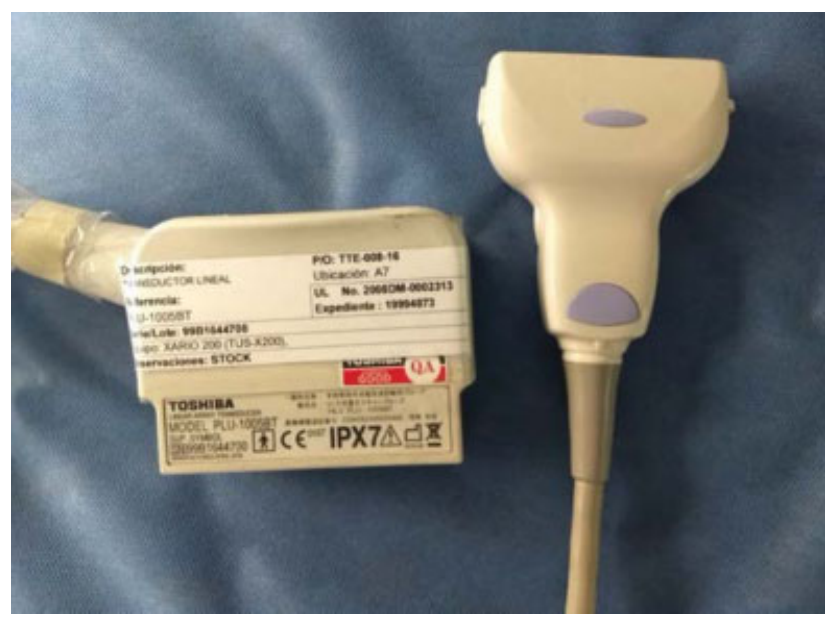

Fig. 2 Transductor lineal empleado para identificar la arteria colateral radial y las perforantes. con el fin de hallar la arteria colateral radial del brazo estudiado, consecuentemente se desplazó el transductor hacia el epicóndilo lateral del húmero. Una vez identificada la arteria, se orientó el transductor en sentido longitudinal al brazo y así se identificó cada una de las perforantes, las cuales se evaluaron para determinar las variables: la edad, el género, el diámetro y la velocidad picosistólica (VPS) de la arteria colateral radial, el tipo de perforante y a su vez, el diámetro y VPS de cada una de las perforantes, la cantidad de perforantes que se encontraron, la distancia de las perforantes al punto de referencia (epicóndilo lateral), la zona anatómica con mayor número de perforantes y las venas acompañantes de las perforantes.

Se realizó un registro fotográfico del Doppler de cada perforante con su respectiva velocidad en el formato de Doppler espectral, por lo demás se procedió al registro de los datos obtenidos en el formato de recolección de datos (-Figura 3 y 4 ).

\section{Análisis estadístico}

Se utilizaron frecuencias, medidas de tendencia central y de dispersión. Su correspondiente análisis estadístico se realizó mediante el programa STATA 13.0.

El estudio fue aprobado por el comité de ética de investigación con seres humanos del Hospital de San José.

\section{Resultados}

Se realizó el estudio doppler color de forma bilateral en los brazos de treinta participantes voluntarios sanos con una edad promedio de $25 \pm 4,9$ años (rango: 20 a 39 años), la mayoría de los participantes fueron mujeres (66,7\%). Utilizando la técnica mencionada anteriormente, se evidenció que el promedio del diámetro de la arteria colateral radial fue $2,47 \pm 0,8 \mathrm{~mm}$ del lado derecho $\mathrm{y}$ $2,59 \pm 0,65 \mathrm{~mm}$ del lado izquierdo y en cuanto al promedio de velocidad de la ACR fue de $36,5 \pm 12,2 \mathrm{~cm} / \mathrm{seg} \mathrm{y}$ $35,18 \pm 17,2 \mathrm{~cm} / \mathrm{seg}$, respectivamente (-Tabla $\mathbf{1}$ ).

Al realizar la búsqueda de las perforantes de la ARC, se encontró un promedio de 3,3 $\pm 0,99$ (rango: 2 a 6 ) perforantes derechos y 3,1 $\pm 0,86$ (rango: 2 a 5) perforantes izquierdas de las cuales la mayoría eran distales $67 \%$ del lado derecho y $62,7 \%$ del lado izquierdo, de igual manera la mayoría de las perforantes tenían un curso musculocutáneo $70 \%$ del lado derecho y 56,3\% del lado izquierdo (Gráfica 1 y 7), con una velocidad promedio de $22,9 \pm 13,4 \mathrm{~cm} / \mathrm{seg}$ del lado derecho y $19,78 \pm 19,78 \mathrm{~cm} / \mathrm{seg}$ de lado izquierdo $\mathrm{y}$ un diámetro promedio de $1,3 \pm 0,39 \mathrm{~mm} \quad$ y $\quad 1,3 \pm 0,35 \mathrm{~mm}$ respectivamente (Tabla 1 ).

La cantidad de perforantes grandes que tenían un diámetro mayor a $1 \mathrm{~mm}$ fue en promedio de 3 del lado derecho y 2 del lado izquierdo en comparación a las perforantes pequeñas que tenían un diámetro menor o igual a $1 \mathrm{~mm}$ con un promedio menor a 1 en ambos brazos. El número de venas acompañantes de las perforantes en la región anterolateral del brazo según los resultados, fue entre 0 venas acompañantes a 1 vena acompañante. No se encontró en ninguno de los casos más de una vena acompañante (Tabla 1). 
94 Mapeo Doppler color de las perforantes de la arteria colateral radial Torres-Fuentes y col

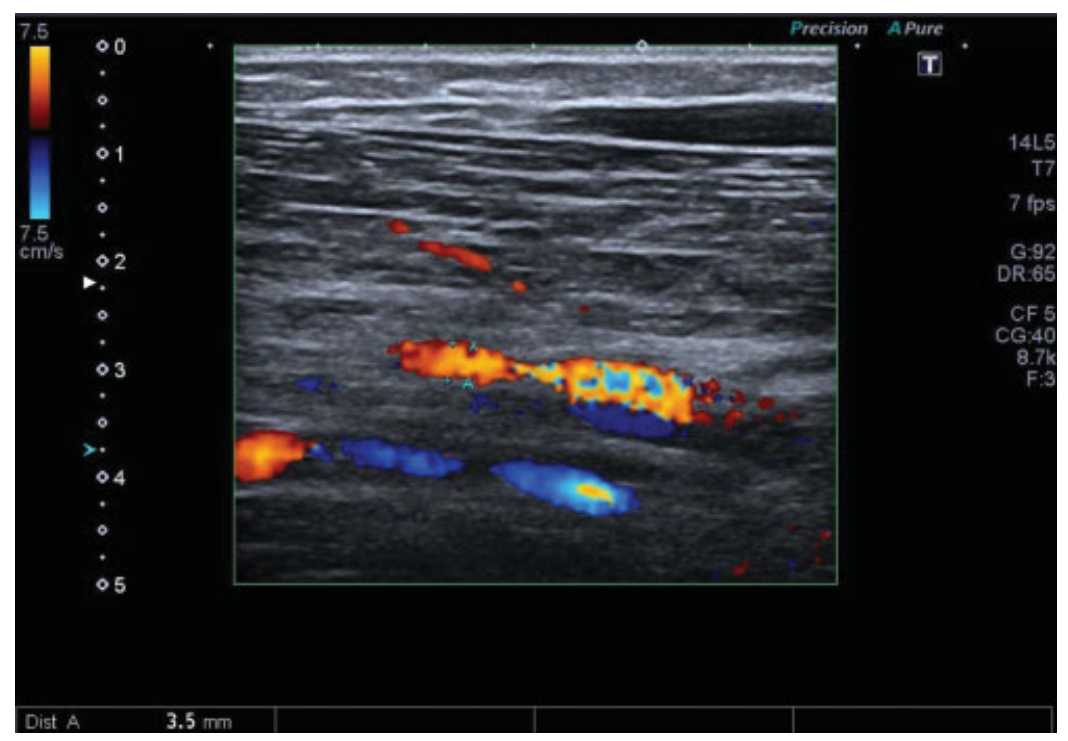

Fig. 3 Ejemplo doppler color de un participante donde se evidencia el diámetro de la Arteria colateral Radial que es de 3,5 mm (límite superior).

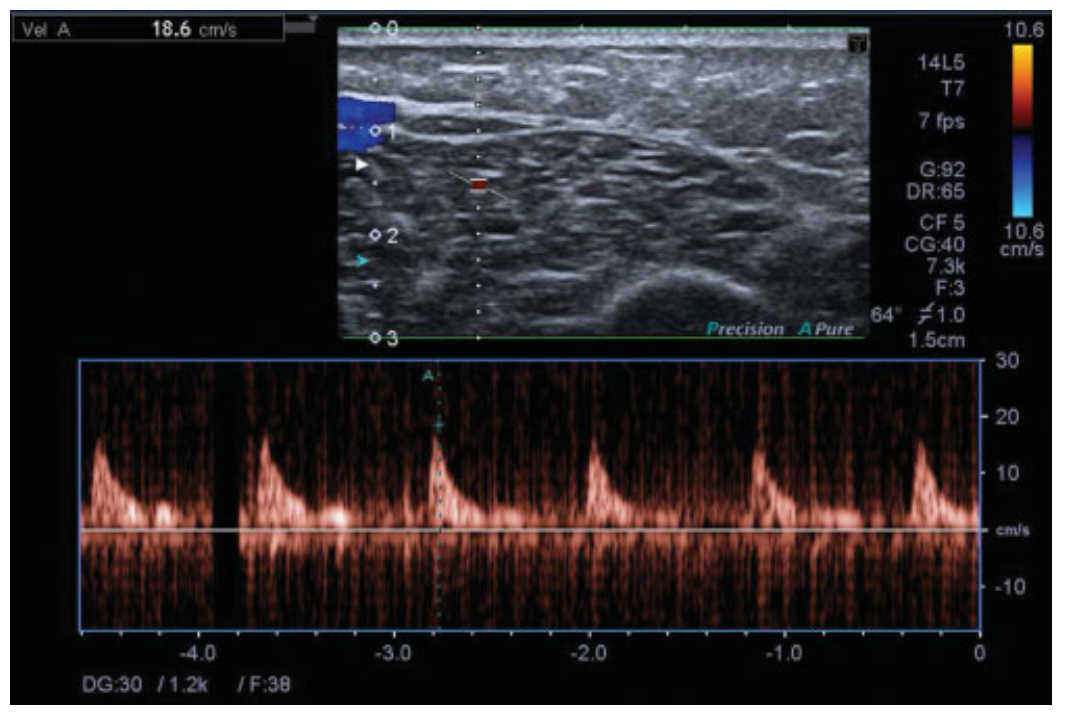

Fig. 4 Ejemplo Doppler color de un participante donde se evidencia la velocidad de una de las perforantes encontradas que es de $18,6 \mathrm{~cm} / \mathrm{seg}$ con un trayecto miocutaneo.

Tabla 1 Resultados obtenidos en miembro superior derecho e izquierdo (Promedio \pm desviación estándar (rango))

\begin{tabular}{|l|l|l|}
\hline & Miembro superior derecho & Miembro superior izquierdo \\
\hline Velocidad VPS ARC (cm/seg) & $36,5 \pm 12,2(11-57,5)$ & $35,1 \pm 17,2(10,7-83,5)$ \\
\hline Diámetro ARC $(\mathrm{mm})$ & $2,47 \pm 0,8(1-4,2)$ & $2,59 \pm 0,65(1,1-3,5)$ \\
\hline Velocidad VPS perforantes $(\mathrm{cm} / \mathrm{seg})$ & $22,9 \pm 13,4(6,1-65)$ & $19,78 \pm 19,7(6,9-55)$ \\
\hline Diámetro perforantes $(\mathrm{mm})$ & $1,3 \pm 0,39(0,5-2,6)$ & $1,3 \pm 0,35(0,7-2,7)$ \\
\hline Perforantes grandes $(>1 \mathrm{~mm})$ & $2,53 \pm 1,54(0-6)$ & $2,43 \pm 1,45(0-5)$ \\
\hline Perforantes pequeñas $(<\mathrm{o}=1 \mathrm{~mm})$ & $0,8 \pm 0,96(0-3)$ & $0,7 \pm 1,02(0-4)$ \\
\hline Venas acompañantes & $0,39 \pm 0,49(0-1)$ & $0,4 \pm 0,49(0-1)$ \\
\hline
\end{tabular}

La distancia de las perforantes encontradas con respecto al epicóndilo lateral fue de $8,05 \pm 3,29 \mathrm{~cm}$ (rango: 1,2 a 15) en el miembro superior derecho y de $7,92 \pm 3,32 \mathrm{~cm}$ (rango: 2 a 17) en el miembro superior izquierdo. Se determinó la moda entre los valores de distancia obtenidos en los miembros superiores, siendo ella de $9 \mathrm{~cm}$, con lo cual se llegó a determinar que el lugar más frecuente de ubicación de esas perforantes en el miembro 


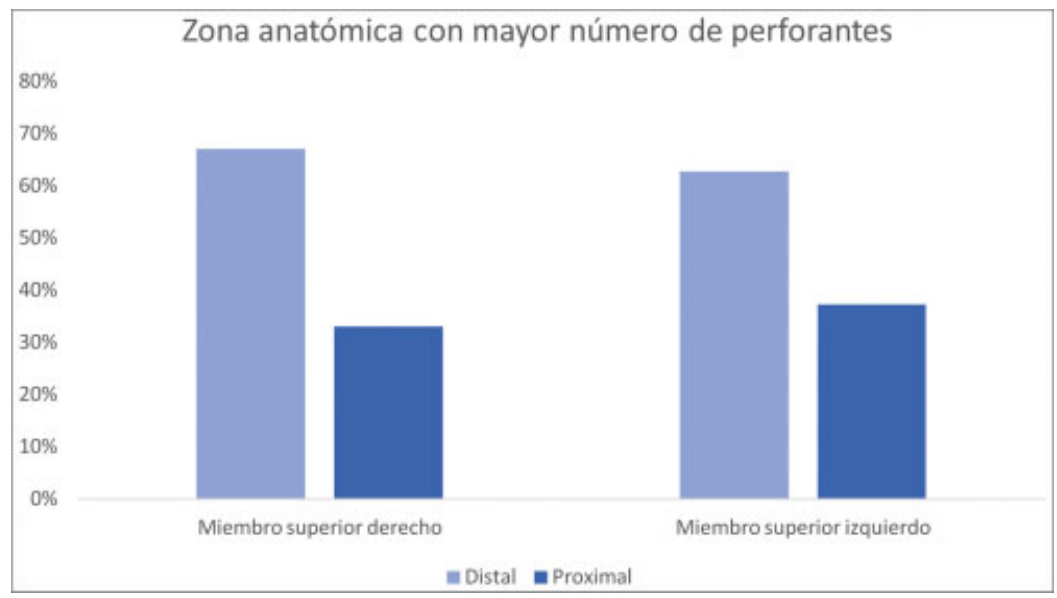

Gráfica 1 Resultados obtenidos en porcentaje de la zona anatómica en el brazo con mayor número de perforantes.

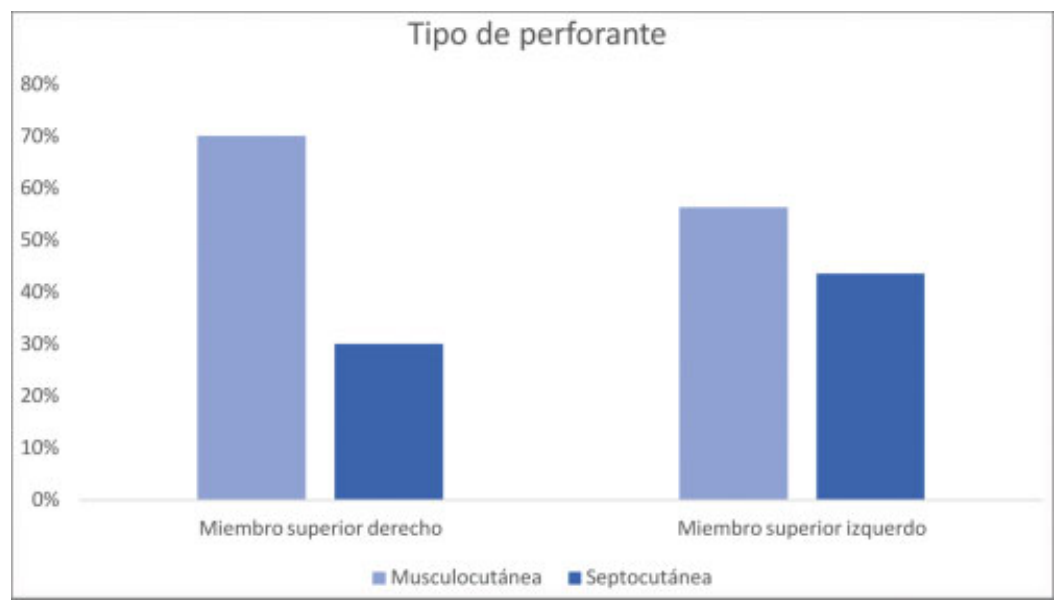

Gráfica 2 Resultados obtenidos en porcentaje del tipo de perforante encontrada. Curso del vaso perforante según las estructuras anatómicas por las que vaya su recorrido.

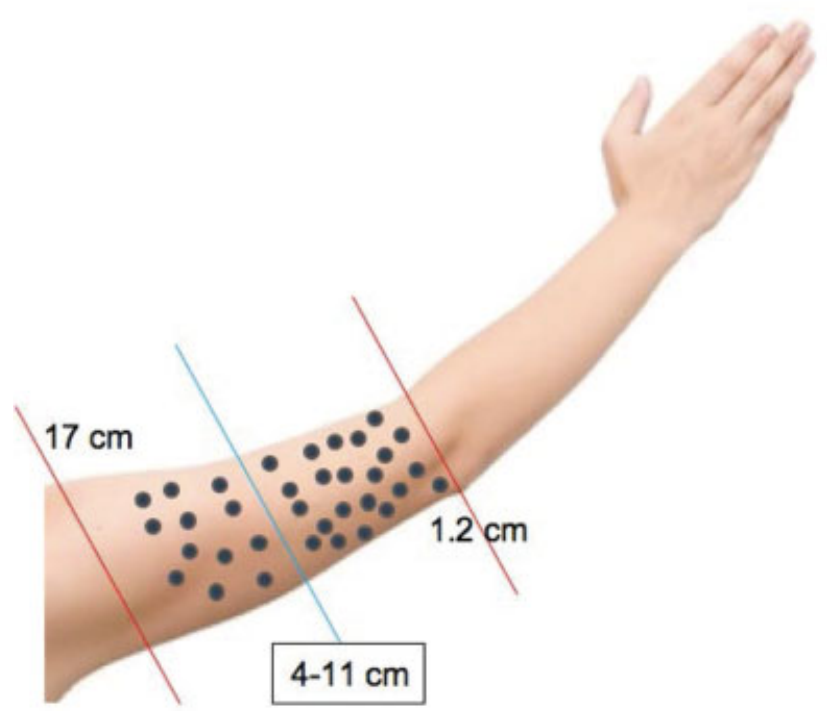

Fig. 5 Gráfica de la zona anatómica en el brazo con mayor número de perforantes con predominio en la región distal de manera constante, tanto en el miembro superior derecho como en el izquierdo. superior, es entre 4 y $11 \mathrm{~cm}$ al epicóndilo lateral, con valores mínimos de $1,2 \mathrm{~cm}$ y máximos de $17 \mathrm{~cm}$ con respecto al epicóndilo (-Figura $\mathbf{5}$ ).

\section{Discusión}

Actualmente hay muy pocos reportes en la literatura, mencionando que, en Colombia, hasta el momento no existen estudios como este. Es importante destacar que la mayoría de los estudios utilizan principalmente la angiotomografía (AngioTAC) para el estudio de las mismas, sin embargo, ante las limitaciones económicas del sistema de salud, sobre todo en nuestro país, utilizar este tipo de herramientas no es tan factible, por eso el presente trabajo, muestra que de una forma más sencilla y económica, se puede obtener la información necesaria acerca del patrón de perforantes y las características de las mismas, así como el diseño de colgajos. Eso es de gran valor, ya que el estudio doppler color se convierte en una herramienta útil como guía en el preoperatorio e intraoperatoria.

Nuestro estudio mostró diferencias con estudios previos respecto a que, la perforantes eptocutánea más distal 
típicamente se ramificaba aproximadamente $4 \mathrm{~cm}$ proximalmente al epicóndilo lateral. ${ }^{10,11}$ Este estudio identificó que las perforantes se ramifican desde $1,2 \mathrm{~cm}$ del epicóndilo lateral hasta $17 \mathrm{~cm}$ del mismo, siendo la mayoría encontradas aproximadamente a $9 \mathrm{~cm}$ de nuestro punto de referencia Además, otro punto importante a tener en cuenta, es que se constató que la mayoría de las perforantes tenían un curso musculocutáneo a diferencia de lo reportado en la literatura.

Se logró determinar además en este estudio, el número de perforantes según su ubicación en mitades, siendo la región distal su lugar más frecuente, y específicamente la zona comprendida entre 4 y $11 \mathrm{~cm}$ del epicóndilo lateral, siendo así, el resultado con mayor importancia ya que con dicho conocimiento se vuelve predecible la ubicación de las perforantes de la arteria colateral radial, facilitando el planeamiento de este tipo de colgajos y por lo tanto haciendo su utilización cada vez más frecuente.

Por otra parte, hay que tener en cuenta que, el doppler logra determinar la velocidad de flujo de cada una de las perforantes, lo cual es de suma importancia para determinar la sobrevida del colgajo, y con el presente trabajo logramos determinar como parámetro velocidades en promedio $22,9 \mathrm{~cm} / \mathrm{s}$ del lado derecho y $19,7 \mathrm{~cm} / \mathrm{s}$ en el lado izquierdo, velocidad que, según nuestra experiencia, es la recomendada para garantizar la sobrevida del colgajo.

En cuanto a las limitaciones que encontramos en este estudio, fue el uso de doppler color dado que es operador dependiente, lo que significa que la práctica y la experiencia del profesional que maneja este método es muy importante para la obtención de resultados confiables, ${ }^{20}$ por tal motivo, la ecografía Doppler Color que se realizó en el grupo de voluntarios sanos, se hizo en una sola intervención y medición, sin seguimiento, y por tal motivo, no hubo necesidad de realizar control de pérdidas en el seguimiento, y además fue realizada por un solo especialista con experiencia del Servicio de Radiología de la institución para evitar la variabilidad interobservador y evitar sesgos de medición.

Dentro de las implicaciones más importantes en la realización del esteestudio fue valorar a través de un método diagnóstico en este caso, el doppler color todas las características de las perforantes de la arteria colateral radial, para posteriormente ser usado en la clínica y realizar ese tipo de colgajo de perforantes en el cubrimiento de defectos en el miembro superior, siguiendo los parámetros encontrados, permitiendo así incrementar el uso de ese tipo de colgajos como una opción segura para el cirujano.

\section{Conclusiones}

Los resultados de este estudio, establecieron la presencia, ubicación, tipo y características anatómicas de los vasos perforantes dependientes de la arteria colateral radial, con un estudio doppler color, donde se identificó su predominio en el área distal del brazo y se describió la VPS de las perforantes encontradas.

Conflicto de Intereses

Los autores del trabajo declaran no tener ningún conflicto de intereses.

\section{Bibliografía}

1 Appleton SE, Morris SF. Anatomy and physiology of perforator flaps of the upper limb. Hand Clin 2014;30(02):123-135, v

2 Morris SF, Tang M, Almutari K, Geddes C, Yang D. The anatomic basis of perforator flaps. Clin Plast Surg 2010;37(04):553-570, xi

3 Chim H, Ng ZY, Carlsen BT, Mohan AT, Saint-Cyr M. Soft tissue coverage of the upper extremity: an overview. Hand Clin 2014;30 (04):459-473, vi

4 Herter F, Ninkovic M, Ninkovic M. Rational flap selection and timing for coverage of complex upper extremity trauma. J PlastReconstrAesthet Surg 2007;60(07):760-768

5 Sinna R, Boloorchi A, Mahajan AL, Qassemyar Q, Robbe M. What should define a "perforator flap"? PlastReconstr Surg 2010;126 (06):2258-2263

6 Bravo FG, Schwarze HP. Free-style local perforator flaps: concept and classification system. J PlastReconstrAesthet Surg 2009;62 (05):602-608, discussion 609

7 D'Arpa S, Toia F, Pirrello R, Moschella F, Cordova A. Propeller flaps: a review of indications, technique, and results. BioMed Res Int 2014;2014:986829

8 Murakami M, Ono S, Ishii N, Hyakusoku H. Reconstruction of elbow region defects using radial collateral artery perforator (RCAP)-based propeller flaps. J PlastReconstrAesthet Surg 2012; 65(10):1418-1421

9 Ono S, Sebastin SJ, Yazaki N, Hyakusoku H, Chung KC. Clinical applications of perforator-based propeller flaps in upper limb soft tissue reconstruction. J Hand Surg Am 2011;36(05):853-863

10 Ninkovic M, Dornseifer U. Lateral arm flap. In: Wei FC, editor. Flaps and reconstructive surgery: Elsevier Inc.; 2009:305-319

11 Sauerbier M, Unglaub F. Perforator flaps in the upper extremity. Clin Plast Surg 2010;37(04):667-676, vii

12 Brunetti B, Tenna S, Segreto F, Del Buono R, Persichetti P. Lateral arm reconstruction with posterior radial collateral artery perforator based flap. J PlastReconstrAesthet Surg 2013;66(06):875-876

13 Tan BK, Lim BH. The lateral forearm flap as a modification of the lateral arm flap: vascular anatomy and clinical implications. PlastReconstr Surg 2000;105(07):2400-2404

14 Pederson WC, Brown EZ. Nonmicrosurgical Coverage of the Upper Extremity. In: Wolfe SW, editor. Green's Operative Hand Surgery. 7th ed: Elsevier Inc.; 2016:1645-1720

15 Jobe MT. Microsurgery. In: Canale T, Beaty MJH, editors. Campbell's Operative Orthopaedics. 12 ed: Elsevier; 2013:3126-3197

16 Tamai S. History of microsurgery. PlastReconstr Surg 2009;124(6, Suppl): e282-e294

17 Sun R, Ding Y, Sun C, et al. Color Doppler Sonographic and Cadaveric Study of the Arterial Vascularity of the Lateral Upper Arm Flap. J Ultrasound Med 2016;35(04):767-774

18 Chaput B, Gandolfi S, Ho Quoc C, Chavoin JP, Garrido I, Grolleau JL. Reconstruction of cubital fossa skin necrosis with radial collateral artery perforator-based propeller flap (RCAP). Ann ChirPlastEsthet 2014;59(01):65-69

19 Kneser U, Beier JP, Schmitz M, et al. Zonal perfusion patterns in pedicled free-style perforator flaps. J PlastReconstrAesthet Surg 2014;67(01):e9-e17

20 Poggio GA, Mariano J, Gopar LA, Ucar ME. La ecografía primero: ¿Por qué, cómo y cuándo? Rev Argent Radiol. 2017;81(03):192-203 